\title{
Brain Tumor Stem Cells
}

\author{
ICHIRO NAKANO, AND HARLEY I. KORNBLUM
}

\author{
Departments of Psychiatry [H.I.K.], Pharmacology [H.I.K.], Neurosurgery [I.N.], and Pediatrics [I.N., HIK.], David Geffen School of \\ Medicine at University of California, Los Angeles, Los Angeles, CA, 90095
}

\begin{abstract}
Cancers are composed of heterogeneous cell populations ranging from highly proliferative immature cells to more differentiated cells of various cell lineages. Recent advances in stem cell research have allowed for the demonstration of the existence of cancer stem cells in acute myeloid leukemia, breast cancer, and, most recently, in brain tumors. Each of these has some similarities with the normal stem cells in the corresponding organs. In brain tumors, putative cancer stem cells have been identified from glioblastoma multiforme, medulloblastoma and ependymoma. These tumorderived cells self-renew under clonal conditions, and differentiate into neuron- and glia-like cells as well as into abnormal cells with mixed phenotypes. The tumor stem cells, but not the rest of tumor cells form secondary tumors by transplantation into immunodeficient mouse brain. In this review, we discuss the cellular and molecular relationships between brain tumor stem cells and normal neural stem cells, and also the possible clinical implications of brain tumor stem cells. (Pediatr Res 59: 54R-58R, 2006)
\end{abstract}

$\mathrm{C}$ ancers, rather than consisting of single cell types, contain a variety of different cells with different phenotypic and genetic characteristics. There are numerous tumor types that can be found within the CNS. Metastatic tumors arise from tissue outside the CNS, and spread hematogenously to the brain. Primary CNS tumors are thought to arise directly from CNS tissue. Generally, these exclude such intracranial tumors as meningioma and acoustic neurinoma that, while existing only within the skull, they are not thought to arise from brain tissue. The most common form of brain tumors in pediatric patients is medulloblastoma, and in adults is glioblastoma multiforme (GBM). Both tumor types have aggressive characteristics, and cause high mortality and morbidity. Medulloblastomas generally occur in the posterior fossa, although metastases can spread throughout the CNS, including socalled "drop mets" in the spinal cord. GBM are the most aggressive form of gliomas, a heterogeneous group of tumors. While medulloblastoma is often treatable, there is still a high rate of morbidity and mortality. To date, GBM remains one of the most difficult cancers to treat, with less than $5 \%$ of $5-y$ survival rate, despite of multiple treatments such as direct surgery, radiation therapy, and chemotherapy. Traditional

Received December 7, 2005; accepted December 21, 2005.

Correspondence: Harley I. Kornblum, M.D., Ph.D., Address for mailing corresponding author and proofs: Departments of Psychiatry, Pharmacology, and Pediatrics, David Geffen School of Medicine at UCLA, Los Angeles, CA 90095; e-mail: hkornblum@mednet.ucla.edu

Some of the work described here was supported by a Jonsson Comprehensive Cancer Center Interdisciplinary Grant.

DOI: $10.1203 / 01 . p d r .0000203568 .63482 . f 9$ thinking holds that any (or many) cells within a tumor will be capable of giving rise to another tumor (1). The cancer stem cell hypothesis dictates that tumors arise from a single, selfrenewing cell type, which then gives rise to the rest of the tumor, including a variety of "more differentiated" cell types. These cancer stem cells would be a minority population within the tumor. The practical implications of this hypothesis is that any curative therapy will need to kill or stop the cancer stem cell from proliferating, whereas therapies targeted at derivatives of the cancer stem cell will not be curative. Studies have provided strong evidence for the cancer stem cell hypothesis in leukemia and in breast cancer $(2,3)$.

The first cancer which was found to contain stem cells was acute myeloid leukemia (AML) (2) (Table 1). A subfraction of cells in AML resembled normal hematopoietic stem cells based upon their morphologic and immunohistochimal characteristics. It was found that this subset of cells, but not the rest of the tumor cells, could form AML when xenotransplanted into immnodeficient mice. The corresponding secondary AML in mice possessed similar histopathological characteristics to the primary tumor. The second cancer type in which caner stem cells were identified was breast cancer (3). In this study, the authors used markers associated with normal ductal stem cells, to positively (CD44) and negatively (CD24) sorted cells. When small numbers of these cells were injected into immunodeficient mice, they formed tumors at very high frequency, while the stem cell negative fraction did not form tumors. These secondary tumors were histologically similar to the primary tumors and also contained a subpopulation of CD44+, CD24- cancer stem cells which could, in turn form tumors in other mice.

In addition to providing the first documentations of cancer stem cells, both of these studies raised the strong possibility that the origin of the cancer stem cells, themselves, lay in the tissue-specific or adult stem cells resident within the tissue. Thus, although the cancer stem cell hypothesis does not require cancer stem cells to be derived from mutations of normal stem cells, there is evidence to suggest that, at least in these two cancers, such is the case.

Abbreviations: bFGF, basic fibroblast growth factor; GBM, glioblastoma multiforme; GFAP, glial fibrillary acidic protein; LIF, leukemia inhibitory factor; PTEN, phosphatase and tenascin homolog; TOR, target of rapamycin 
Table 1. Organ-specific cancer stem cells

\begin{tabular}{|c|c|c|c|}
\hline Organ & Malignancy & Related normal stem cell & Surface markers \\
\hline Bone marrow & Acute myeloid leukemia & Hematopoietic stem cell & $\mathrm{CD}^{2+}$ \\
\hline Mammary gland & Breast cancer & $\begin{array}{l}\text { Adult mammary epithelial } \\
\text { stem cell }\end{array}$ & $\begin{array}{l}\mathrm{CD} 44^{+} \\
\mathrm{CD} 24^{-/ \text {low }}\end{array}$ \\
\hline
\end{tabular}

Tissue specific cancer stem cells and strategy to identify them.

\section{NEURAL STEM CELLS AND BRAIN TUMOR STEM CELLS}

Neural stem cells are a form of tissue-specific stem cell. They are self-renewing and capable of forming the major cell types intrinsic to the brain, neurons, astrocytes and oligodendrocytes. Neural stem cells were originally isolated in culture by exposing dissociated CNS cells to epidermal growth factor (EGF) in a relatively minimal medium (4). As a result, large spheres of cells formed from single cells which could then be re-dissociated and seeded at extremely low density to produce new spheres, indicating a capacity for self-renewal. In the proliferative and undifferentiated state, these cells express the intermediate filament, nestin. However, when EGF is removed, the cells begin to express markers typical of differentiated neurons and glia. Thus, these cells meet the criteria of being stem cells in that they are both self-renewing and multipotent.

Neural stem cells can be isolated from the CNS at virtually any stage of development after neural tube closure, and possibly before, through adulthood $(4,5)$. In vivo, neural stem cells are generally reside within specialized germinal zones situated along the surface of the neural tube-the developing and mature ventricular system. However, there is some heterogeneity of cells that share the critical features of neural stem cells; self-renewal and multipotency (6). During development, neural stem cells divide rapidly and extensively self-renew. Some investigators call these cells multipotent progenitors, since, in general, stem cells are thought as relatively slowly dividing. Radial glia, the bipolar cells extending from the ventricular to the pial surface of the developing neural tube have been long perceived as "simple" guides for migrating cortical neurons. Several recent studies, however, have demonstrated that radial glia, themselves function as neural stem cells during prenatal rodent development. Later, in postnatal stages and adulthood, a type of neural stem cell exists that divides relatively slowly and produces more rapidly dividing self-renewing progenitors which, in turn, produces more committed progenitors. Recent evidence has indicated that these putative, slowly dividing cells express glial fibrillary acidic protein (GFAP), a classic marker for astrocytes $(7,8)$. Likewise, in the adult human brain, neural stem cells are also GFAP-positive cells (9).

Neural stem cells have been isolated and enriched from fetal and adult human brain and grown as neurospheres and adherent cultures. Human neural stem cells are CD133positive and CD24-negative (10). Flow sorting for these markers, greatly enriches cultures for neurosphere-forming cells, although this set of markers does not completely purify neural stem cells.

Following the isolation of neural stem cells, there was wide speculation that mutations of neural stem cells were the origins of many brain tumors. There are several lines of evidence supporting the hypothesis that brain tumors arise from aberrant neural stem cell proliferation (11) including the fact that many brain tumors contain neuronal and glial elements and express the intermediate filament nestin (12). Direct evidence for a neural stem cell origin of human brain tumors is difficult to obtain. However, an elegant series of studies using transgenic models has demonstrated that nestinexpressing progenitors overexpressing the oncogene, c-Myc, can give rise to gliomas and medulloblastomas $(13,14)$. Transformation of GFAP positive cells in vivo can give rise to gliomas, as well (15). In the past, this finding would have been taken as evidence of astrocytes being the origin of these experimental tumors, but now, given that postnatal neural stem cells are known to be GFAP positive (8), these results are also consistent with a neural stem cell origin.

Despite evidence that neural stem cells can be the source of brain tumors under some experimental conditions, it is unlikely that they are the source of all brain tumors. Medulloblastomas are tumors of the cerebellum. Recent microarray and animal model studies strongly indicate that the source of many, if not all medulloblastomas is the external granule cell (16). This cell type, like neural stem cells, is self-renewing, but it is not multipotent under most conditions. Additionally, many tumors appear to have origins away from the ventricular surface, the site of neural stem cells in vivo, making it more likely that they arise from transformation of other progenitors or more fully differentiated glia. One potential cell of origin could be the recently identified glial-like cells with a capacity to self-renew and produce neurons, astrocytes and oligodendrocytes that are dispersed throughout the cortex and cerebellum, at sites distant from the ventricular surface (17).

Despite the fact that not all brain tumors are likely to be derived from neural stem cells, many brain tumors do contain stem cells within them that bear many similarities to neural stem cells. We found that several brain tumors, including astrocytomas (low and high grade), ependymoma, glioblastoma multiforme, and medulloblastomas contained cells that proliferated in the presence of EGF, basic fibroblast growth factor (bFGF) and leukemia inhibitory factor (LIF) under conditions identical to those reported for embryonic human neural stem cells (18). Others have also obtained similar data with gliomas $(19,20)$ medulloblastomas (20) and ependymo- 
mas (21). These cells readily formed neurospheres as shown in Fig. 1. Immunocytochemical analysis of these tumor-derived spheres demonstrated that most expressed nestin (Fig. 1). Following growth factor withdrawal, most tumor-derived spheres gave rise to cells with glial and neuronal morphology and antigenic characteristics (Fig. 1). Tumor-derived spheres could be passaged clonally, and these clones could again be demonstrated to be multipotent. The numbers of neuron-like and glial-like cells in each clone within a tumor were remarkably similar although the relative proportions varied greatly from tumor to tumor (18).

The data described above demonstrate the presence of self-renewing, multipotent progenitors within brain tumors, but do not prove that they are cancer stem cells. Two studies from different groups, however, do demonstrate this. Galli et al. (22) demonstrated that neurosphere cultures generated from GBM cells (in a manner very similar to Hemmati et al. (18)) could give rise to GBMs in immunodeficient mice. Tumors could result from cultures that had been expanded from single cells and bore the same cytogenetic abnormalities as the parent tumors. Singh et al. (23) used a slightly different strategy. They sorted glioblastomas and medulloblastomas for CD133 and found that the CD133- positive cells transplanted at low density readily formed tumors in immunodeficient mice. These tumors could be re-sorted for CD133 positive cells, which could then form new tumors in recipient mice. CD133 negative cells, even at much higher concentrations, did not form tumors. More recently, tumorigenic stem cells were isolated from ependymomas from various brain and spinal cord regions. Interestingly, these cells had the properties of radial glia. Taken together, the studies of Singh et al. $(20,23)$ Galli et al. (22) and our own study (18) strongly support the hypothesis that at least some brain tumors contain cancer stem cells with at least some of the characteristics of neural stem cells.

\section{CRITICAL REGULATOR CANDIDATES OF SELF-RENEWAL OF BRAIN TUMOR STEM CELLS}

The isolation of cancer stem cells from brain tumors is important because of the implications that only through attacking these cells, will we be able to eradicate the tumors that contain them. As stated above, cancer stem cells and adult stem cells share the properties of self-renewal and multipotentiality. Several studies have demonstrated that genes known to play roles in the self-renewal of somatic stem cells also are likely to play similar roles in cancer stem cells. Some of these pathways, which are not mutually exclusive, are described here.

Bmi-1. Bmi-1 is a polycomb transcription factor and is known to regulate chrmomatin remodeling. Bmi-1 has been previously shown to regulate the proliferation of hematopoetic stem cells as well as leukemic stem cells (24). Analysis of Bmi-1 mutant mice have shown that it is also important for self-renewal of normal neural stem cells (25). Bmi-1 binds to p14INKa and p16ARF, and inhibits the activation of their signaling (26). These two downstream genes are highly expressed in primary GBM as well as oligodendrocytoma, another type of glioma. The high level of bmi-1 expression in our tumor-derived progenitors in spheres (18) suggests that this gene, as well as its signaling pathway, plays an important role in the proliferation of not only GBM but also stem cells in GBM. Recently bmi-1 and its associated genes were found as critical indicators of the prognosis of various cancers including glioma, according to the cDNA microarray data set (27). This also suggests that bmi- 1 and the genes that it regulates have a strong influence on proliferation of tumor cells.

PTEN and the PI3 Kinase-Akt pathway. Activation of PI3 kinase leads to phosphoryolation of Akt which, in turn, leads to promotion of proliferation and cell survival, among other things (28). A part of this cascade includes TOR (target of

\section{Neural Stem Cells and Brain Tumor Stem Cells}

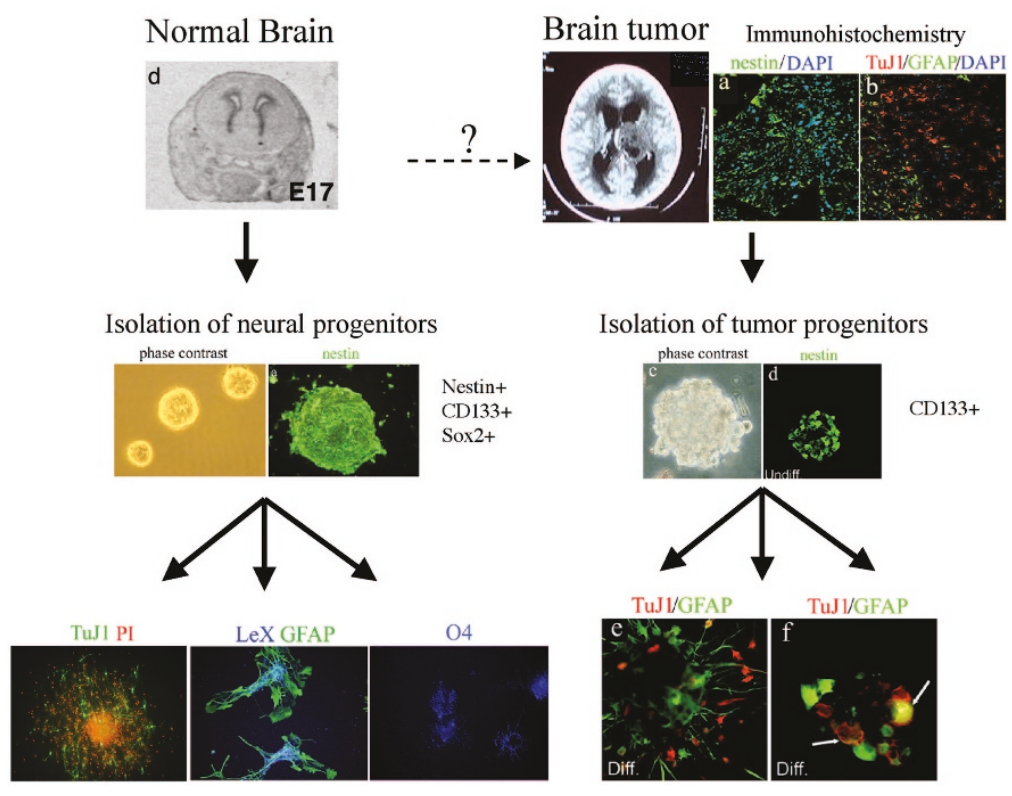

Figure 1. Characteristics of brain tumor stem/progenitor cell. Normal neural stem cells (left panel) can be enriched as neurospheres in culture, and self-renew. Majority of cells in proliferating neurospheres are nestin-positive. Once these spheres are induced to differentiate, they form TuJ1-positive neurons, GFAP-positive astrocytes, as well as O4-positive oligodendrocytes. Brain tumors (right panel) contain heterogeneous cell populations. Immunohistochemistry shows multiple cell types labeled with various markers such as nestin, TuJ1, and GFAP $(A, B)$. Similar to the normal stem cells, brain tumor stem cells can be isolated and enriched as spheres $(C)$, which are highly expressing nestin $(D)$. Besides with their self-renewal capacity, they also differentiate into multiple cell types, which exist in the original tumor, such as TuJ1-positive neuron-like cells $(E)$, GFAP-positive astrocyte-like cells $(E)$, as well as double positive (arrows in $F$ ) abnormal cells. 
rapamycin). Many tumors frequently have enhanced activity of this pathway. Mutations of the EGF receptor, for example, are frequently found in GBM and confer a worse prognosis with a more aggressive tumor. Another mechanism by which this pathway is regulated is via the phosphatase PTEN (phosphatase and tenascin homolog deleted on chromosome 10). PTEN suppresses Akt phosphorylation by reversing PI3 kinase-driven phosphorylation. PTEN is a tumor suppressor gene, and is frequently deleted in GBM. We have previously demonstrated that PTEN is a critical regulator of neural stem cell self-renewal, as its loss induces greater proliferation as well as enhanced recruitment of neural stem cells from a quiescent to an active cycling state $(29,30)$.

Drugs that interact with this pathway are potential candidates for treatment of brain tumors. Inhibitors of EGFR activation have been recently shown to be effective in treating patients with the EGFR VIII mutation (31), an active form of the receptor. Rapamycin is a drug that inhibits TOR and is under investigation for a variety of cancers, including GBM. It is unknown, however, whether these agents selectively inhibit cancer stem cell proliferation.

Maternal embryonic leucine-zipper kinase (MELK). MELK encodes a serine/threonine kinase with a leucinezipper domain, and is a poorly characterized member of snf1/AMPK family. Several members of this family are known as tumor survival factors under conditions of nutrient starvation. Our previous studies demonstrated that normal neural stem cells highly express MELK. Functional analysis demonstrated that MELK regulates the self-renewal of murine (32) and human (unpublished) neural stem cells. MELK was recently identified by microarray analysis to be highly correlated with malignancy of a variety of tumors and to be an important regulator of cell cycle progression in these cancer cells (33). We have found that MELK is highly expressed in brain tumors as well as in brain tumor stem cell containing cultures (18). Functional studies in these cultures indicate that MELK regulates the proliferation of both putative cancer stem cells as well as non stem cell components (unpublished). The mechanism by which MELK regulates proliferation in cancer stem cells or other cells is still unknown. Previous studies have suggested that one mechanism of MELK action is by regulated the transcription factor B-Myb, a known cell cycle regulator (32). However, our own preliminary data suggest that this is not the only function of MELK. MELK has both kinase and RNA processing functions, both of which could contribute to its net effects.

Wnt and Hedgehog signaling in medulloblastomas. As described above, cancer stem cells have been reported for medulloblastomas, although there is some disagreement in the literature about whether these cells represent true stem cells. The Wnt and sonic hedgehog signaling cascades promote the proliferation of numerous cells, including granule cell progenitors of the cerebellum, the presumed cells of origin of medulloblastoms. Both have also been directly shown to regulate the growth of murine and human medulloblastomas (16). Mutations of the sonic hedgehog receptor patched (which functions to inhibit the pathway) results in the formation of medullo- blastomas in both mice and humans. It remains to be seen whether these effects are on a cancer stem cell, per se.

\section{CLINICAL SIGNIFICANCE OF THE CANCER STEM CELL THEORY}

The isolation of cancer stem cells is an important step in the understanding of tumor biology. However, there are also practical implications in the diagnosis and treatment of brain for the clinician.

Significance for brain tumor stratification. Traditional anatomic/pathologic categorization of tumors have very limited ability to completely stratify patients into meaningful subgroups for prognosis and intervention. Protein expression by immunohistochemistry has greatly enhanced the potential link between pathologic diagnosis and prognosis. However, relative lack of useful antibodies shows the limitation of this strategy. Recently, large scale gene expression study by microarray and analyses of specific pathways have been more successful at regrouping patients within broad histologic categories (16). The existence of caner stem cells raises the possibility that expression profiling and molecular pathway analysis of these cells will provide further useful stratification of tumors. For example, one might isolate cancer stem cells from individual patients and then analyze gene or protein expression profiles as well as the activity of the specific pathways described above (Fig. 2).

Significance for brain treatment. The isolation and demonstration of brain tumor stem cells, suggests that, for those tumors that contain such cells, treatment can only be gauged as successful if the stem cell component is successfully eradicated (Fig. 2). Since, by their nature, stem cells are heartier and more resistant to insult, this may prove to be a daunting task. There are several potential avenues to address this issue, however. First, since cancer stem cells may use specific molecular pathways to drive their self-renewal, one might use known and as-yet-to-be-discovered selective inhibitors of these pathways to attack them. In fact, as intimated above, two groups of agents, EGF receptor inhibitors and rapamycinrelated compounds, that are already in clinical trial and showing some promise are predicted to inhibit cancer stem cell self-renewal (31). Another way to attack cancer stem cells

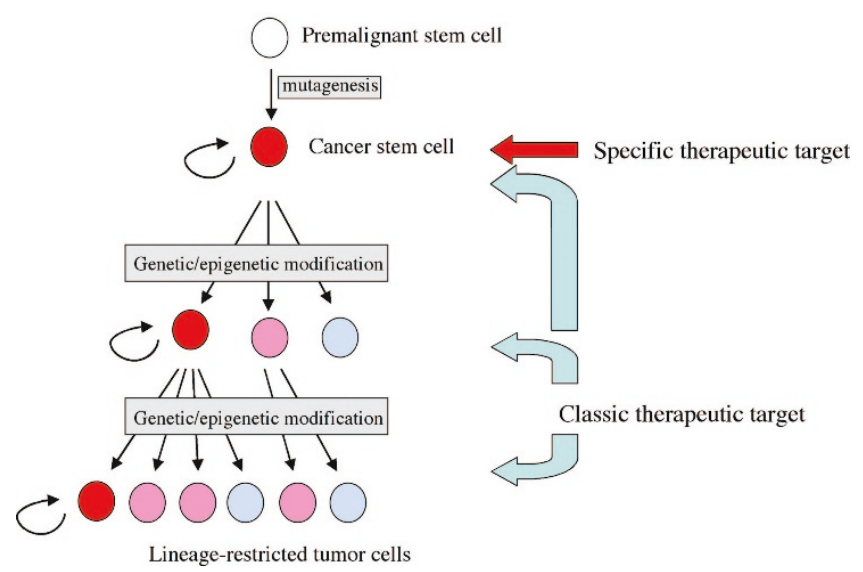

Figure 2. Clinical implication for cancer stem cell theory. 
would be to use them as targets for small molecule screens, an approach that we are taking. Finally, if specific antigens are expressed by cancer stem cells, then these antigens could be used in immunotherapies, which have already shown some promise in the treatment of glioma.

Are there implications for neural repair. Neural stem and progenitor cells are potential sources of therapy for a wide variety of disorders, ranging from enzymatic deficiency to neurodegenerative disease. It is well known that transplantation of pluripotent embryonic stem cells into the brain or other tissue can result in teratoma formation. The possibility that neural stem and progenitor cells can give rise to tumors theoretically raises the possibility that transplantation of the cells could result in tumor formation. It is important to state, however, that this simply has not been shown to be the case, at least in the short to moderate timespans used in animal studies. To our knowledge, despite more than one thousand reports of neural stem and progenitor cell transplantation, none have reported malignant transformation.

\section{CONCLUSIONS}

The isolation and initial characterization of brain tumor stem cells provides hope that new avenues of therapy will be opened up. However, many questions remain before our fully utilizing these discoveries. Among these are the following: Do all brain tumors contain cancer stem cells? What are the differences and similarities between cancer stem cells from different tumors? Does malignant progression of low grade to high grade gliomas directly involve brain tumor stem cells? Is CD-133 the best marker for the prospective isolation of cancer stem cells? Can we attack brain tumor stem cells without attacking normal neural stem cells? Would this matter? These questions and many others highlight the early state of this field and the great amount of work needed before putting the discovery of cancer stem cells to productive use.

\section{REFERENCES}

1. Reya T, Morrison SJ, Clarke MF, Weissman IL 2001 Stem cells, cancer, and cancer stem cells. Nature 414:105-111

2. Bonnet D, Dick JE 1997 Human acute myeloid leukemia is organized as a hierarchy that originates from a primitive hematopoietic cell. Nat Med 3:730-737

3. Al-Hajj M, Wicha MS, Benito-Hernandez Morrison SJ, Clarke MF 2003 Prospective identification of tumorigenic breast cancer cells. Proc Natl Acad Sci U S A 100:3983-3988

4. Reynolds BA, Weiss S 1992 Generation of neurons and astrocytes from isolated cells of the adult mammalian central nervous system. Science 255:1707-1710

5. Weiss S, Dunne C, Hewson J, Wohl C, Wheatley M, Peterson AC, Reynolds BA 1996 Multipotent CNS stem cells are present in the adult mammalian spinal cord and ventricular neuroaxis. J Neurosci 16:7599-7609

6. Hitoshi S, Tropepe V, Ekker M, van der Kooy D 2002 Neural stem cell lineages are regionally specified, but not committed, within distinct compartments of the developing brain. Development 129:233-244

7. Doetsch F, Caille I, Lim DA, Garcia-Verdugo JM, Alvarez-Buylla A 1999 Subventricular zone astrocytes are neural stem cells in the adult mammalian brain. Cell 97:703-716

8. Imura T, Kornblum HI, Sofroniew MV 2003 The predominant neural stem cell isolated from postnatal and adult forebrain but not early embryonic forebrain expresses GFAP. J Neurosci 23:2824-2832

9. Sanai N, Tramontin AD, Quinones-Hinojosa A, Barbaro NM, Gupta N, Kunwar S, Lawton MT, McDermott MW, Parsa AT, Manuel-Garcia Verdugo J, Berger MS, Alvarez-Buylla A 2004 Unique astrocyte ribbon in adult human brain contains neural stem cells but lacks chain migration. Nature 427:740-744
10. Uchida N, Buck DW, He D, Reitsma MJ, Masek M, Phan TV, Tsukamoto AS, Gage FH, Weissman IL 2000 Direct isolation of human central nervous system stem cells. Proc Natl Acad Sci U S A 97:14720-14725

11. Oliver TG, Wechsler-Reya RJ 2004 Getting at the root and stem of brain tumors Neuron 42:885-888

12. Rorke LB 1997 Pathologic diagnosis as the gold standard. Cancer 79:665-667

13. Rao G, Pedone CA, Coffin CM, Holland EC, Fults DW 2003 c-Myc enhances sonic hedgehog-induced medulloblastoma formation from nestin-expressing neural progenitors in mice. Neoplasia 5:198-204

14. Rao G, Pedone CA, Valle LD, Reiss K, Holland EC, Fults DW 2004 Sonic hedgehog and insulin-like growth factor signaling synergize to induce medulloblastoma formation from nestin-expressing neural progenitors in mice. Oncogene 23:6156-6162

15. Uhrbom L, Dai C, Celestino JC, Rosenblum MK, Fuller GN, Holland EC 2002 Ink4a-Arf loss cooperates with KRas activation in astrocytes and neural progenitors to generate glioblastomas of various morphologies depending on activated Akt. Cancer Res 62:5551-5558

16. Pomeroy SL, Tamayo P, Gaasenbeek M, Sturla LM, Angelo M, McLaughlin ME, Kim JY, Goumnerova LC, Black PM, Lau C, Allen JC, Zagzag D, Olson JM, Curran T, Wetmore C, Biegel JA, Poggio T, Mukherjee S, Rifkin R, Califano A, Stolovitzky G, Louis DN, Mesirov JP, Lander ES, Golub TR 2002 Prediction of central nervous system embryonal tumour outcome based on gene expression. Nature 415:436-442

17. Nunes MC, Roy NS, Keyoung HM, Goodman RR, McKhann G 2nd, Jiang L, Kang J, Nedergaard M, Goldman SA 2003 Identification and isolation of multipotential neural progenitor cells from the subcortical white matter of the adult human brain. Nat Med 9:439-447

18. Hemmati HD, Nakano I, Lazareff JA, Masterman-Smith M, Geschwind DH, Bronner-Fraser M, Kornblum HI 2003 Cancerous stem cells can arise from pediatric brain tumors. Proc Natl Acad Sci U S A 100:15178-15183

19. Ignatova TN, Kukekov VG, Laywell ED, Suslov ON, Vrionis FD, Steindler DA 2002 Human cortical glial tumors contain neural stem-like cells expressing astroglial and neuronal markers in vitro. Glia 39:193-206

20. Singh SK, Clarke ID, Terasaki M, Bonn VE, Hawkins C, Squire J, Dirks PB 2003 Identification of a cancer stem cell in human brain tumors. Cancer Res 63:58215828

21. Taylor MD, Poppleton H, Fuller C, Su X, Liu Y, Jensen P, Magdaleno S, Dalton J, Calabrese C, Board J, Macdonald T, Rutka J, Guha A, Gajjar A, Curran T, Gilbertson RJ 2005 Radial glia cells are candidate stem cells of ependymoma. Cancer Cell 8:323-335

22. Galli R, Binda E, Orfanelli U, Cipelletti B, Gritti A, De Vitis S, Fiocco R, Foroni C, Dimeco F, Vescovi A 2004 Isolation and characterization of tumorigenic, stem-like neural precursors from human glioblastoma. Cancer Res 64:7011-7021

23. Singh SK, Hawkins C, Clarke ID, Squire JA, Bayani J, Hide T, Henkelman RM, Cusimano MD, Dirks PB 2004 Identification of human brain tumour initiating cells. Nature 432:396-401

24. Park IK, Qian D, Kiel M, Becker MW, Pihalja M, Weissman IL, Morrison SJ, Clarke MF 2003 Bmi-1 is required for maintenance of adult self-renewing haematopoietic stem cells. Nature 423:302-305

25. Molofsky AV, Pardal R, Iwashita T, Park IK, Clarke MF, Morrison SJ 2003 Bmi-1 dependence distinguishes neural stem cell self-renewal from progenitor proliferation. Nature 425:962-967

26. Molofsky AV, He S, Bydon M, Morrison SJ, Pardal R 2005 Bmi-1 promotes neural stem cell self-renewal and neural development but not mouse growth and survival by repressing the p16Ink4a and p19Arf senescence pathways. Genes Dev 19:14321437

27. Glinsky GV, Berezovska O, Glinskii AB 2005 Microarray analysis identifies a death-from-cancer signature predicting therapy failure in patients with multiple types of cancer. J Clin Invest 115:1503-1521

28. Sinor AD, Lillien L 2004 Akt-1 expression level regulates CNS precursors. J Neurosci 24:8531-8541

29. Groszer M, Erickson R, Scripture-Adams DD, Lesche R, Trumpp A, Zack JA Kornblum HI, Liu X, Wu H 2001 Negative regulation of neural stem/progenitor cell proliferation by the Pten tumor suppressor gene in vivo. Science 294:2186-2189

30. Groszer M, Erickson R, Scripture-Adams DD, Dougherty JD, Le Belle J, Zack JA, Geschwind DH, Liu X, Kornblum HI, Wu H 2005 PTEN negatively regulates neural stem cell self-renewal by modulating G0-G1 cell cycle entry. Proc Natl Acad Sci U S A Dec 22; [Epub ahead of print]

31. Mellinghoff IK, Wang MY, Vivanco I, Haas-Kogan DA, Zhu S, Dia EQ, Lu KV, Yoshimoto K, Huang JH, Chute DJ, Riggs BL, Horvath S, Liau LM, Cavenee WK, Rao PN, Beroukhim R, Peck TC, Lee JC, Sellers WR, Stokoe D, Prados M, Cloughesy TF, Sawyers CL, Mischel PS 2005 Molecular determinants of the response of glioblastomas to EGFR kinase inhibitors. N Engl J Med 353:2012-2024

32. Nakano I, Paucar AA, Bajpai R, Dougherty JD, Zewail A, Kelly TK, Kim KJ, Ou J, Groszer M, Imura T, Freije WA, Nelson SF, Sofroniew MV, Wu H, Liu X, Terskikh AV, Geschwind DH, Kornblum HI 2005 Maternal embryonic leucine zipper kinase (MELK) regulates multipotent neural progenitor proliferation. J Cell Biol 170:413-427

33. Gray D, Jubb AM, Hogue D, Dowd P, Kljavin N, Yi S, Bai W, Frantz G, Zhang Z, Koeppen H, de Sauvage FJ, Davis DP 2005 Maternal embryonic leucine zipper kinase/murine protein serine-threonine kinase 38 is a promising therapeutic target for multiple cancers. Cancer Res 65:9751-9761 